\title{
Myeloid-Related Protein Activity in Rheumatoid Arthritis
}

\author{
Charles J. Malemud \\ Division of Rheumatic Diseases, Department of Medicine, University Hospitals Case Medical Center, Case Western Reserve University \\ School of Medicine, 2061 Cornell Road, Suite 207, Cleveland, OH 44106, USA
}

Correspondence should be addressed to Charles J. Malemud, cjm4@cwru.edu

Received 14 March 2011; Revised 12 May 2011; Accepted 23 June 2011

Academic Editor: Rosario Scalia

Copyright () 2011 Charles J. Malemud. This is an open access article distributed under the Creative Commons Attribution License, which permits unrestricted use, distribution, and reproduction in any medium, provided the original work is properly cited.

SA100A8, SA100A9, and SA100A12 are members of the myeloid-related protein class. SA100A8 and SA100A9, also known as MRP-8 and MRP-14, respectively, are intracellular $\mathrm{Ca}^{2+}$-binding proteins produced mainly by neutrophils and monocytes where they exist as a heterodimeric complex in the cytosol. The MRP-8/-14 complex has been shown to promote chronic inflammation associated with rheumatoid arthritis (RA). In that regard, MRP-8 and MRP-14 regulate the inflammatory response through their capacity to recruit neutrophils and monocytes to target tissues resulting in attachment to endothelium. MRPs also activate the signal transduction pathway principally involving the stress-activated/mitogen-activated protein kinases. MRP-8/MRP-14 also increased nitric oxide synthesis. Most recently, the MRP-8/MRP-14 complex was shown to be a novel ligand for the toll-like receptors (TLRs) and TLR-4, in particular. Engagement of TLRs by the MRP-8/-14 complex may be particularly important for activating antigen-presenting dendritic cells which regulate critical autoimmune responses that promote chronic synovitis characteristic of RA.

\section{Introduction}

The myeloid-related protein (MRP) family of proteins include MRP-8, also known as S100A8, MRP-14, also known as SA100A9, and S100A12 [1]. MRP-8 and MRP-14 are intracellular $\mathrm{Ca}^{2+}$-binding proteins that are produced by a variety of myeloid cells. MRP-8 and MRP-14 exist as a heterodimeric complex in the cytosol of polymorphonuclear leukocytes and monocytes $[2,3]$.

\section{The MRP Complex and Inflammation}

MRPs have been implicated as important contributors to inflammation in general, and to the overall inflammatory response associated with autoimmune disorders such as rheumatoid arthritis (RA) [4, 5]. The MRP-8/14 complex, in particular, has been proposed to play a critical role in regulating several of the inflammatory responses associated with RA because both MRP-8 and MRP-14 can promote chronic inflammation and act to recruit neutrophils and monocytes to inflamed tissue by enhancing their migration, retention, and attachment to the endothelium [6]. In this regard, the best evidence for the fundamental role played by the MRPs in inflammation $[7,8]$ can be gleaned from the results of experimental studies which demonstrated that exogenously added MRP-8/14 was capable of directly inducing macrophage recruitment to inflamed tissues which was also accompanied by increased levels of nitric oxide (NO) $[8,9]$.

\section{The MRP Complex, NO, and Signal Transduction}

NO has been shown to be an important soluble mediator of inflammatory responses in adult RA via the upregulation of inducible nitric oxide synthase (iNOS) as a result of nuclear factor $\kappa \mathrm{B}(\mathrm{NF}-\kappa \mathrm{B})$ activation [10]. In addition, $\mathrm{NO}$ has been shown to be a potent inducer of apoptosis in cultured articular chondrocytes $[11,12]$. Importantly, MRP-8/14-mediated up-regulation of iNOS gene expression by macrophages in vitro was also shown to also be accompanied by the activation (i.e., phosphorylation) of multiple protein kinase-mediated signal transduction pathways, including those involving cJun-N-amino-terminal kinase (JNK), extracellular-regulated kinase 1/2 (ERK1/2), and Janus kinase/signal transducers 
and activators of transcription (JAK/STAT) as well as activation of NF- $\kappa$ B. Interestingly, JAK/STAT was found to be preferentially activated by interferon- $\gamma$ (IFN- $\gamma$ ) rather than by MRP-8/14 [4]. However, the activation of multiple downstream signal transduction pathways by NO, IFN- $\gamma$, MRP-8/14, and other proinflammatory cytokines, namely, tumor necrosis factor- $\alpha$ (TNF- $\alpha$ ), IL-6, IL-8, IL-12/23, IL-18, and so forth [13] has profound implications for any future therapeutic interventions for RA [14-17] because it would be unlikely that suppression or inhibition of any one of these signaling pathways would ameliorate the inflammation of RA or reduce articular cartilage destruction and/or subchondral bone erosions characteristic of RA [18].

\section{Tissue Localization and Circulating MRP Complex in RA}

MRP antigens were found to reside only in the macrophage subtype in the synovial membrane of RA synovial tissue [4]. Importantly, the relevance of MRP antigen localization to synovial joint cartilage destruction and subchondral bone erosion in RA was also supported by the finding that MRP antigens were localized only to those cells that were adjacent to the cartilage-pannus junction and only in RA patients with clinically active disease [4]. These findings suggested the possibility that suppression of MRP gene expression might be a suitable novel target for therapeutic intervention to inhibit the progression of juvenile chronic arthritis $[19,20]$ and/or adult RA [8].

Importantly, in RA patients, the serum levels of MRP8/14 and SA100A12 correlated with one another [5]. Moreover, serum levels of MRP-8/14 and S100A12 in 138 patients with RA were substantially elevated compared to 44 healthy controls. The S100A9 serum levels were detected using an anti-MRP-14 antibody that not only measured MRP-14 but also detected MRP-14 in its complex with MRP-8. These levels were about 100 -fold lower than those reported in other studies [21, 22], and the differences between the results of these two studies $[5,21]$ could be accounted for on the basis of the specificity of the anticalprotectin (an antibody generated against the MRP-8/14 complex) that was previously employed [21, 22]. Taken together, these results indicated that elevated levels of circulating serum MRP antigens in RA patients were coordinately increased. Thus, the level of circulating MRP-8/14/SA100A12 could potentially be employed as a biomarker to track the pathologic and radiographic progression of RA as well as clinical responses and other objective measurements to approved RA therapeutic DMARDs and/or biologic agents.

In addition to the above, elevated levels of these MRP antigens were also shown to significantly contribute to vascular injury and thus to the peripheral end-organ damage in adult RA [5] which both significantly contribute to the numerous comorbid components of RA disease activity. The elevated levels of peripheral and organ-related MRP antigens may also be associated with precocious atheroma formation and the accelerated development of atherosclerosis which has been found to correlate with vascular injury and the later development of coronary artery disease [23, 24] which is often seen as a significant comorbidity in RA patients with clinically active disease [25-27]. Interestingly, carotid intima media thickness (cIMT), a marker of preclinical atherosclerosis, and flow-mediated dilatation (FMD) of the brachial artery, a marker of endothelial dysfunction, were assessed in 79 patients with early RA and in 44 age-matched and sex-matched controls [28]. Importantly, the FMD and cIMT at baseline were not significantly different between the two groups. Thus, the results of this study indicated that although preclinical evidence of atherosclerosis was often associated with early RA, there was also a strong indication that early therapeutic intervention in RA (perhaps by targeting MRP-8/14 antigens) could establish a means to significantly reduce the progression of atherosclerosis in these RA patients.

Importantly, in systemic-onset juvenile RA (JRA), a strong and positive correlative relationship was found between the level of MRP-8/14 in the peripheral circulation and the level of another proinflammatory cytokine, germane to RA disease activity and progression, namely, interleukin$1 \beta$ (IL-1 $\beta$ ), [29]. This result suggested that in JRA, the MRP-8/14 complex in conjunction with IL- $1 \beta$ constituted a novel positive feedback pathway that could account for the robustness of macrophage activation in this arthritic disorder of childhood.

\section{What Is the Evidence That the MRPs Help Drive RA Disease Progression?}

5.1. Toll-Like Receptors (TLRs). TLR-mediated signaling is a crucial component in driving both innate and adaptive immune activation [30]. Recently, the MRP-8/14 complex was shown to be a novel ligand for the TLR pathway and for TLR-4, in particular [31, 32]. Thus, in a fashion similar to LPS, a known activator of TLR-mediated signaling, MRP-8 induced the hyperphosphorylation of IL-1 receptorassociated kinase- 1 in human IFN- $\gamma$-primed monocytes that was also characterized by elevated levels of TNF- $\alpha$, IL$1 \beta$, and IL-12 [32]. In addition, LPS and MRP-8 were used to determine the extent to which these responses occurred in murine phagocytes without a functional TLR4 complex. Thus, compared to their wild-type counterparts, TLR-4 mutant mice demonstrated a similar lack of response to both LPS and MRP-8, but in contrast to wild-type mice, stimulation of TLR- $9,-8$, or -7 with $\mathrm{cpG}$ and R848, respectively, revealed no differences in response. These data indicated that MRP-8 was an endogenous ligand of TLR-4 and that the MRP-8/-14 complex stimulation of TLR- 4 was a mechanism for increasing the effect of LPS stimulated by the MRP-8/14 complex. In this regard, the TLR pathway having been engaged by the MRP-8/14 complex may be particularly important for activating antigen-presenting dendritic cells which regulate, in part, several of the autoinflammatory and autoimmune responses, which in the context of RA promotes the chronic state of synovitis [32]. 
5.2. Chemokines and Adhesion Molecules. The elevated production of chemokines and adhesion molecules are also crucial to the pathogenesis of experimentally-induced murine arthritis [33] and human RA [34]. Thus, TNF- $\alpha$ not only promotes the inflammatory responses associated with RA [34] but also enhances the migration of neutrophils towards CCL3, known as macrophage inhibitory protein-1 $\alpha$ (MIP$1 \alpha)$, via its capacity to interact with CCR5 $[34,35]$.

The MIP- $1 \alpha /$ CCR 5 response also appears to be a consequence of TNF- $\alpha$-induced up-regulation of CD11b/CD18, also known as macrophage-activating complex-1, (Mac1 ), which was found on the surface of neutrophils. In this regard, Newton and Hogg [36] found that MRP-14 activated Mac-1 on the surface of neutrophils, whereas Rouleau et al. [37] showed that SA100A12 induced enhanced neutrophil migration and adhesion as well as release from mouse bone marrow at concentrations comparable to those levels of SA100A12 found in the plasma of patients with inflammatory arthritis. Therefore, it was noteworthy that BIIL3, a long-acting leukotriene B4 (LTB4) receptor antagonist, inhibited LTB4-induced Mac-1 expression in patients with RA [38].

\subsection{MRP Levels in Response to Antirheumatic Disease Ther-} apies. Although a rapid lowering of MRP levels in synovial tissue sublining macrophages was associated with the positive clinical response of RA patients to anti-TNF$\alpha$ therapy [39, 40], could MRP levels be employed as a sensitive biomarker for distinguishing between effective and ineffective drug interventions? In this regard, the data from 2 randomized, controlled RA clinical trials were reexamined in which experimental therapies designed to neutralize either monocyte chemotactic protein-1 (MCP1) activity or the interaction between $\mathrm{C} 5 \mathrm{a}$ and its receptor C5aR were assessed with each of these strategies failing to show clinical efficacy. In this analysis, Wijbrandts et al. [41] combined the data from these 2 studies with clinical response and CD68 data from other RA clinical trials in which combinations of DMARDs and biological agents resulted in a positive clinical response. The "standardized response mean" (SRM) was employed as the outcomes measure. The results of this study showed a strong relationship between lower disease activity score-28 (DAS-28) levels (a standard for measuring clinical response in RA clinical trials) and the number of CD68-positive sublining macrophages (SRM, $>0.8$ ). This result indicated that the number of CD68positive sublining macrophages which had been previously shown to be positive for MRP [39] was a sensitive biomarker to measure clinical responses to effective therapies in RA clinical trials, but not for the interventions which were ineffective (anti-MCP-1, SRM, 0.19; anti-C5aR, SRM, -0.071). Furthermore, the difference in the weighted mean of the SRM between clinically effective and ineffective interventions was significant for both DAS-28 and the number of CD68 sublining macrophages which indicated that the weighted mean of the SRM could be used to detect differences between effective and ineffective interventions as well as placebo effects [41]. It should be noted that Haringman et al.
[42] had also previously shown a similar strong correlation between lowered DAS-28 scores and the number of CD68 sublining macrophages (Pearson correlation, $0.874, \mathrm{P}<0.01$ ) in response to several types of antirheumatic therapies.

Additionally, patients with spondyloarthropathy or adult RA who responded favorably to the anti-TNF- $\alpha$ monoclonal antibody infliximab showed a rapid and marked decrease in the level of MRP-positive macrophages in tissue infiltrates, but not in the total population of resident macrophages [43]. Furthermore, infliximab effectively reduced the soluble form of MRP-8/14 in the sera of RA patients but had no effect on the level of the soluble form of CD163 [39].

It has also been important to assess the extent to which the number of MRP-positive macrophages in RA patients in response to anti-TNF- $\alpha$ therapy correlated with changes in other biomarkers of inflammation. To address this issue, van Kuijk et al. [44] showed that lower DAS-28 scores could be achieved in patients with psoriatic arthritis receiving the anti-TNF- $\alpha$ monoclonal antibody, adalimumab compared to placebo. In this analysis of psoriatic arthritis, patients who favorably responded to adalimumab significantly reduced levels of matrix metalloproteinase-3 (MMP-3) and MMP-13, and a trend towards lower levels of von Willerbrand factor, IL-1 and IL-6, was found. Moreover, clinical improvement in these psoriatic arthritis patients in response to adalimumab was strongly correlated with reduced numbers of CD3/CD4positive T cells, and MRP-8-positive macrophages.

Taken together, these results suggested that suppression of Mac-1 expression or blockade of TNF- $\alpha$-mediated cellular signaling by anti-TNF- $\alpha$ therapies may result in the inhibition of migration and adhesion of neutrophils and macrophages into inflamed tissue, in part, by regulating MRP complex-mediated migration and adhesion of these inflammatory cells to specific end-organ sites. Thus, measurements of MRP- 8 was one of several biomarkers, including MMP-3, MMP-13, and CD68 (a marker for macrophages expressing MRP-8) but not CD163 (a marker for resident tissue macrophages) in the sublining of synovial tissue as well as neutrophils. In effect, changes in the levels of these biomarkers were shown to be useful in making predictions regarding the clinical responses of patients with spondyloarthropathy to infliximab [45] but were somewhat in conflict with the results reported by De Rycke et al. [43] who determined that anti-TNF- $\alpha$ therapy appeared to alter the expression of cell-specific CD163 as well as CD68.

\subsection{Regulation of Signal Transduction Pathways: Cell Survival} and Apoptosis. Signal transduction pathways that are activated in RA in response to elevated levels of proinflammatory cytokines $[13,18,46]$ include JNK, ERK 1/2, p38 kinase, and JAK/STAT which may all be differentially activated by the MRP complex [4]. In that regard, Morel et al. [47] recently demonstrated that JNK activity was essential for regulating the phosphorylation of $\mathrm{Mcl}-1$ (a member of the $\mathrm{Bcl} 2$-related protein family) by glycogen synthase kinase 3 (GSK3). Importantly, activation of Mcl-1 may also be responsible for controlling the survival and/or apoptosis of neutrophils, macrophages, and other cells essential in 
activating the inflammation cascade in RA. Thus, in future studies, it will be of interest to determine the extent to which the MRP protein complex regulates the activation of $\mathrm{Mcl}-1$ and GSK3.

Finally, MRP-14 was shown to be a potent activator of p38 kinase-dependent functional responses in intact human neutrophils that included the regulation of neutrophil survival and apoptosis [48]. Thus, suppression of MRP-14 activity may be important in the context of developing novel therapeutic strategies for regulating neutrophil-associated survival in RA via suppression of MRP complex-mediated signaling.

\section{References}

[1] A. Rammes, J. Roth, M. Goebeler, M. Klempt, M. Hartmann, and C. Sorg, "Myeloid-related protein (MRP) 8 and MRP14, calcium binding proteins of the S100 family, are secreted by activated monocytes via a novel, tubulin-dependent pathway," Journal of Biological Chemistry, vol. 272, no. 14, pp. 94969502, 1997.

[2] D. F. Stroncek, R. A. Shankar, and K. M. Skubitz, "The subcellular distribution of myeloid-related protein (MRP8) and MRP14 in human neutrophils," Journal of Translational Medicine, vol. 3, article 36, 2005.

[3] P. A. Hessian and L. Fisher, "The heterodimeric complex of MRP-8 (S100A8) and MRP-14 (S100A9). Antibody recognition, epitope definition, and the implications for structure," European Journal of Biochemistry, vol. 268, no. 2, pp. 353-363, 2001.

[4] P. Youssef, J. Roth, M. Frosch et al., "Expression of myeloid related proteins (MRP) 8 and 14 and the MRP8/14 heterodimer in rheumatoid arthritis synovial membrane," Journal of Rheumatology, vol. 26, no. 12, pp. 2523-2528, 1999.

[5] Y. S. Chen, W. Yan, C. L. Geczy, M. A. Brown, and R. Thomas, "Serum levels of soluble receptor for advanced glycation end products and of S100 proteins are associated with inflammatory, autoantibody, and classical risk markers of joint and vascular damage in rheumatoid arthritis," Arthritis Research and Therapy, vol. 11, no. 2, article R39, 2009.

[6] M. Frosch, A. Strey, T. Vogl et al., "Myeloid-related proteins 8 and 14 are specifically secreted during interaction of phagocytes and activated endothelium and are useful markers for monitoring disease activity in pauciarticular-onset juvenile rheumatoid arthritis," Arthritis and Rheumatism, vol. 43, no. 3, pp. 628-637, 2000.

[7] J. Roth, T. Vogl, C. Sorg, and C. Sunderkötter, "Phagocytespecific S100 proteins: a novel group of proinflammatory molecules," Trends in Immunology, vol. 24, no. 4, pp. 155-158, 2003.

[8] D. Foell, H. Wittkowski, T. Vogl, and J. Roth, "S100 proteins expressed in phagocytes: a novel group of damage-associated molecular pattern molecules," Journal of Leukocyte Biology, vol. 81, no. 1, pp. 28-37, 2007.

[9] P. Pouliot, I. Plante, M. A. Raquil, P. A. Tessier, and M. Olivier, "Myeloid-related proteins rapidly modulate macrophage nitric oxide production during innate immune response," Journal of Immunology, vol. 181, no. 5, pp. 3595-3601, 2008.

[10] G. S. Firestein, "Evolving concepts of rheumatoid arthritis," Nature, vol. 423, no. 6937, pp. 356-361, 2003.

[11] C. J. Malemud, N. Islam, and T. M. Haqqi, "Pathophysiological mechanisms in osteoarthritis lead to novel therapeutic strategies," Cells Tissues Organs, vol. 174, no. 1-2, pp. 34-48, 2003.

[12] C. J. Malemud and H. J. Gillespie, "The role of apoptosis in arthritis," Current Rheumatology Reviews, vol. 1, no. 1, pp. 131142, 2005.

[13] C. J. Malemud and E. Pearlman, "Targeting JAK/STAT signaling pathway in inflammatory diseases," Current Signal Transduction Therapy, vol. 4, no. 3, pp. 201-221, 2009.

[14] L. Sênolt, J. Vencovský, K. Pavelka, C. Ospelt, and S. Gay, "Prospective new biological therapies for rheumatoid arthritis," Autoimmunity Reviews, vol. 9, no. 2, pp. 102-107, 2009.

[15] M. G. Feely, A. Erickson, and J. R. O'Dell, “Therapeutic options for rheumatoid arthritis," Expert Opinion on Pharmacotherapy, vol. 10, no. 13, pp. 2095-2106, 2009.

[16] C. J. Malemud, "Suppression of autoimmune arthritis by small molecule inhibitors of the JAK/STAT pathway," Pharmaceuticals, vol. 3, no. 5, pp. 1446-1455, 2010.

[17] B. A. Wisler, J. E. Dennis, and C. J. Malemud, "New organspecific pharmacological strategies interfering with signaling pathways in inflammatory disorders/autoimmune disorders," Current Signal Transduction Therapy, vol. 6, no. 2, pp. 279291, 2011.

[18] C. J. Malemud, "Dysfunctional immune-mediated inflammation in rheumatoid arthritis dictates that development of anti-rheumatic disease drugs target multiple intracellular signaling pathways," Anti-Inflammatory \& Anti-Allergy Agents in Medicinal Chemistry, vol. 10, pp. 78-84, 2011.

[19] N. M. Wulffraat, P. J. Haas, M. Frosch et al., "Myeloid related protein 8 and 14 secretion reflects phagocyte activation and correlates with disease activity in juvenile idiopathic arthritis treated with autologous stem cell transplantation," Annals of the Rheumatic Diseases, vol. 62, no. 3, pp. 236-241, 2003.

[20] A. Adams and T. J. A. Lehman, "Update on the pathogenesis and treatment of systemic onset juvenile rheumatoid arthritis," Current Opinion in Rheumatology, vol. 17, no. 5, pp. 612616, 2005.

[21] H. B. Hammer, S. Odegard, M. K. Fagerhol et al., "Calprotectin (a major leucocyte protein) is strongly and independently correlated with joint inflammation and damage in rheumatoid arthritis," Annals of the Rheumatic Diseases, vol. 66, no. 8, pp. 1093-1097, 2007.

[22] T. M. Madland, M. Hordvik, H. J. Haga, R. Jonsson, and J. G. Brun, "Leukocyte protein calprotectin and outcome in rheumatoid arthritis. A longitudinal study," Scandinavian Journal of Rheumatology, vol. 31, no. 6, pp. 351-354, 2002.

[23] D. A. Morrow, Y. Wang, K. Croce et al., "Myeloid-related protein $8 / 14$ and the risk of cardiovascular death of myocardial infarction after an acute coronary syndrome in the Pravastatin and Atorvastatin evaluation and infection therapy: thrombolysis in Mycocardial Infarction (PROVE-IT-TIMI-22) trial," American Heart Journal, vol. 155, no. 1, pp. 49-55, 2008.

[24] K. Croce, H. Gao, Y. Wang et al., "Myeloid-related protein$8 / 14$ is critical for the biological response to vascular injury," Circulation, vol. 120, no. 5, pp. 427-436, 2009.

[25] L. de Groot, M. D. Posthumus, C. G. M. Kallenberg, and M. Bijl, "Risk factors and early detection of atherosclerosis in rheumatoid arthritis," European Journal of Clinical Investigation, vol. 40, no. 9, pp. 835-842, 2010.

[26] M. T. Nurmohamed, "The increased cardiovascular risk in rheumatoid arthritis: when does it start?" Arthritis Research \& Therapy, vol. 12, no. 5, p. 140, 2010.

[27] R. J. Bisoendial, E. S. Stroes, and P. P. Tak, "Critical determinants of cardiovascular risk in rheumatoid arthritis," Current Pharmaceutical Design, vol. 17, no. 1, pp. 21-26, 2011. 
[28] A. Södergren, K. Karp, K. Boman et al., "Atherosclerosis in early rheumatoid arthritis: very early endothelial activation and rapid progression of intima media thickness," Arthritis Research \& Therapy, vol. 12, no. 4, p. R158, 2010.

[29] M. Frosch, M. Ahlmann, T. Vogl et al., "The myeloid-related proteins 8 and 14 complex, a novel ligand of toll-like receptor 4 , and interleukin- $1 \beta$ form a positive feedback mechanism in systemic-onset juvenile idiopathic arthritis," Arthritis and Rheumatism, vol. 60, no. 3, pp. 883-891, 2009.

[30] C. Brikos and L. A. O'Neill, "Signalling of toll-like receptors," Handbook of Experimental Pharmacology, no. 183, pp. 21-50, 2008.

[31] T. Vogl, K. Tenbrock, S. Ludwig et al., "Mrp8 and Mrp14 are endogenous activators of Toll-like receptor 4, promoting lethal, endotoxin-induced shock," Nature Medicine, vol. 13, no. 9, pp. 1042-1049, 2007.

[32] Y. Tamaki, Y. Takakubo, T. Hirayama et al., "Expression of Toll-like receptors and their signaling pathways in rheumatoid synovitis," Journal of Rheumatology, vol. 38, no. 5, pp. 810-820, 2011.

[33] G. H. W. Hersmann, J. Kriegsmann, J. Simon, C. Hüttich, and R. Bräuer, "Expression of cell adhesion molecules and cytokines in murine antigen-induced arthritis," Cell Communication and Adhesion, vol. 6, no. 1, pp. 69-82, 1998.

[34] C. J. Malemud and S. K. Reddy, "Targeting cytokines, chemokines and adhesion molecules in rheumatoid arthritis," Current Rheumatology Reviews, vol. 4, no. 4, pp. 219-234, 2008.

[35] F. Montecucco, S. Steffens, F. Burger et al., "Tumor necrosis factor- $\alpha$ (TNF- $\alpha)$ induces integrin CD11b/CD18 (Mac-1) up-regulation and migration to the CC chemokine CCL3 (MIP-1 $\alpha$ ) on human neutrophils through defined signalling pathways," Cellular Signalling, vol. 20, no. 3, pp. 557-568, 2008.

[36] R. A. Newton and N. Hogg, "The human S100 protein MRP-14 is a novel activator of the $\beta 2$ integrin Mac- 1 on neutrophils," Journal of Immunology, vol. 160, no. 3, pp. 14271435, 1998.

[37] P. Rouleau, K. Vandal, C. Ryckman et al., "The calciumbinding protein S100A12 induces neutrophil adhesion, migration and release from bone marrow in mouse at concentrations similar to those found in human inflammatory arthritis," Clinical Immunology, vol. 107, no. 1, pp. 46-54, 2003.

[38] R. Alten, E. Gromnica-Ihle, C. Pohl et al., "Inhibition of leukotriene B4-induced CD11B/CD18 (Mac-1) expression by BIIL 284, a new long acting LTB4 receptor antagonist, in patients with rheumatoid arthritis," Annals of the Rheumatic Diseases, vol. 63, no. 2, pp. 170-176, 2004.

[39] T. J. M. Smeets, M. C. Kraan, M. E. Van Loon, and P. P. Tak, "Tumor necrosis factor $\alpha$ blockade reduces the synovial cell infiltrate early after initiation of treatment, but apparently not by induction of apoptosis in synovial tissue," Arthritis and Rheumatism, vol. 48, no. 8, pp. 2155-2162, 2003.

[40] J. A. Hamilton and P. P. Tak, "The dynamics of macrophage lineage populations in inflammatory and autoimmune diseases," Arthritis and Rheumatism, vol. 60, no. 5, pp. 1210-1221, 2009.

[41] C. A. Wijbrandts, C. E. Vergunst, J. J. Haringman, D. M. Gerlag, T. J. M. Smeets, and P. P. Tak, "Absence of changes in the number of sublining macrophages after ineffective treatment for rheumatoid arthritis: implications for use of synovial sublining macrophages as a biomarker," Arthritis and Rheumatism, vol. 56, no. 11, pp. 3869-3871, 2007.
[42] J. J. Haringman, D. M. Gerlag, A. H. Zwinderman et al., "Synovial tissue macrophages: a sensitive biomarker for response to treatment in patients with rheumatoid arthritis," Annals of the Rheumatic Diseases, vol. 64, no. 6, pp. 834-838, 2005.

[43] L. De Rycke, D. Baeten, D. Foell et al., "Differential expression and response to anti-TNF $\alpha$ treatment of infiltrating versus resident tissue macrophage subsets in autoimmune arthritis," Journal of Pathology, vol. 206, no. 1, pp. 17-27, 2005.

[44] A. W. R. van Kuijk, D. M. Gerlag, K. Vos et al., "A prospective, randomised, placebo-controlled study to identify biomarkers associated with active treatments in psoriatic arthritis: effects of adalimumab treatment on synovial tissue," Annals of the Rheumatic Diseases, vol. 68, no. 8, pp. 1303-1309, 2009.

[45] E. Kruithof, L. De Rycke, B. Vandooren et al., "Identification of synovial biomarkers of response to experimental treatment in early-phase clinical trials in spondyloarthritis," Arthritis and Rheumatism, vol. 54, no. 6, pp. 1795-1804, 2006.

[46] C. J. Malemud, "Differential activation of JAK enzymes in rheumatoid arthritis and autoimmune disorders by proinflammatory cytokines-potential drug targets," International Journal of Interferon, Cytokine and Mediator Research, vol. 2, no. 1, pp. 97-111, 2010.

[47] C. Morel, S. M. Carlson, F. M. White, and R. J. Davis, "Mcl-1 integrates the opposing actions of signaling pathways that mediate survival and apoptosis," Molecular and Cellular Biology, vol. 29, no. 14, pp. 3845-3852, 2009.

[48] G. Lominadze, M. J. Rane, M. Merchant, J. Cai, R. A. Ward, and K. R. McLeish, "Myeloid-related protein-14 is a p38 MAPK substrate in human neutrophils," Journal of Immunology, vol. 174, no. 11, pp. 7257-7267, 2005. 


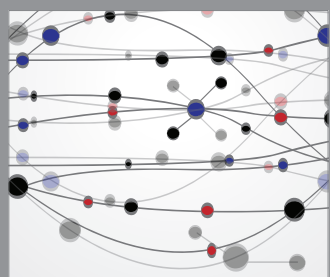

The Scientific World Journal
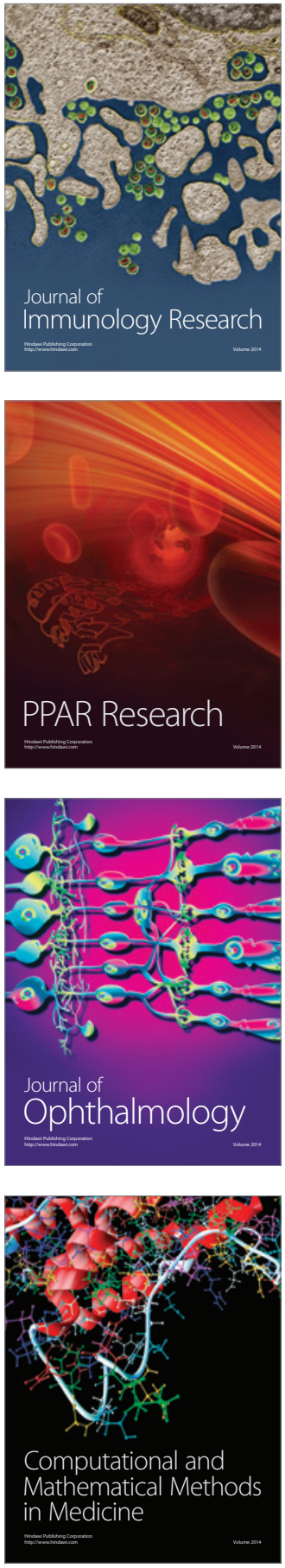

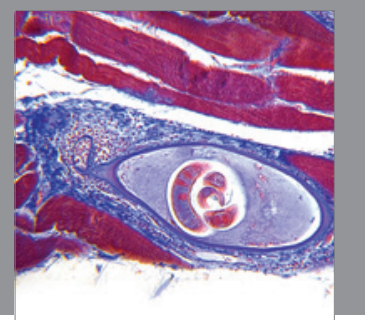

Gastroenterology

Research and Practice
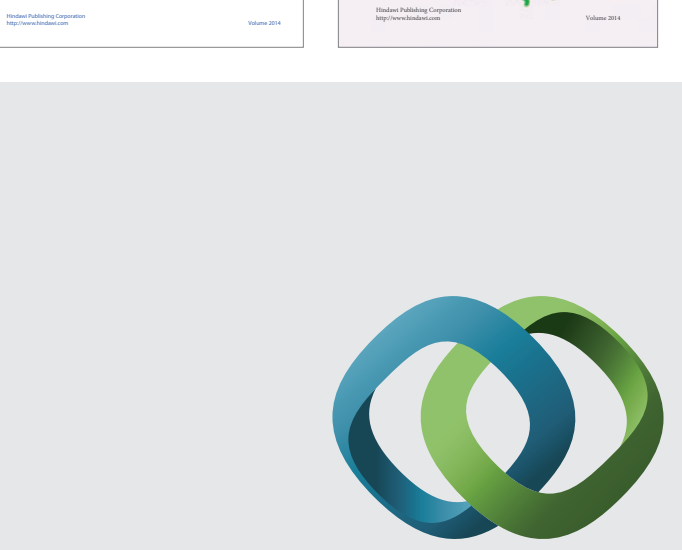

\section{Hindawi}

Submit your manuscripts at

http://www.hindawi.com
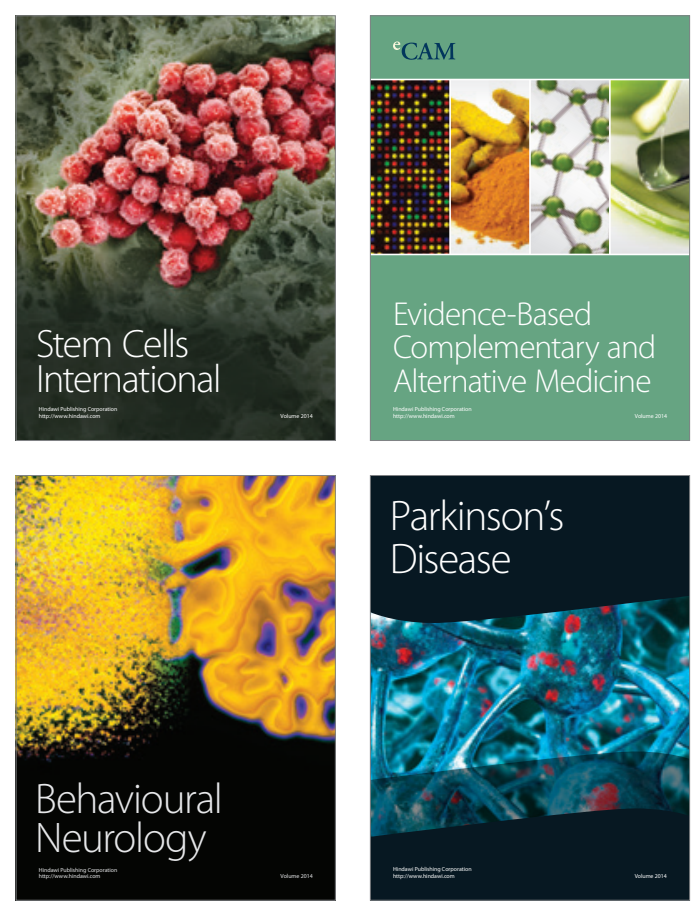

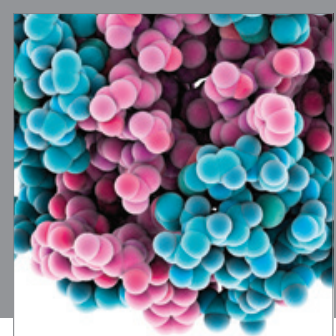

Journal of
Diabetes Research

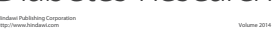

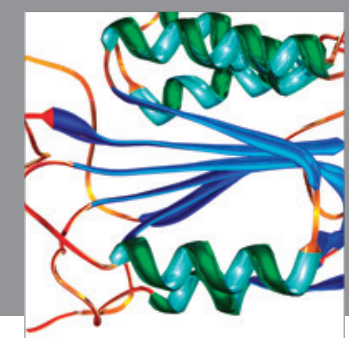

Disease Markers
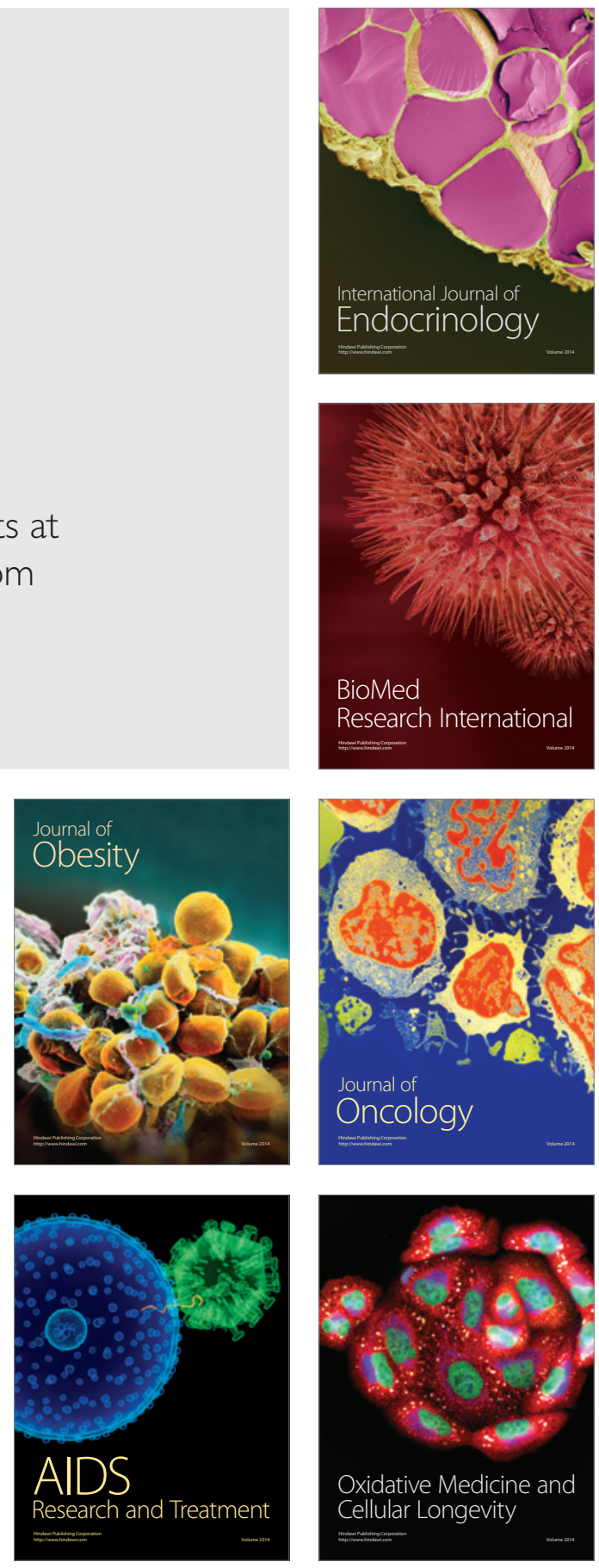